\title{
Line segment Hausdorff distance on face matching
}

\author{
Yongsheng Gao*, Maylor K.H. Leung \\ School of Computer Engineering, Nanyang Technological University, Singapore 639798, Singapore
}

Received 17 March 2000; received in revised form 28 November 2000; accepted 28 November 2000

\begin{abstract}
A novel concept of line segment Hausdorff distance is proposed in this paper. Researchers apply Hausdorff distance to measure the similarity of two point sets. It is extended here to match two sets of line segments. The new approach has the advantage to incorporate structural and spatial information to compute the similarity. The added information can conceptually provide more and better distinctive capability for recognition. This would strengthen and enhance the matching process of similar objects such as faces. The proposed technique has been applied on line segments generated from the edge maps of faces with encouraging result that supports the concept experimentally. The results also implicate that line segments could provide sufficient information for face recognition. This might imply a new way for face coding and recognition. (C) 2001 Pattern Recognition Society. Published by Elsevier Science Ltd. All rights reserved.
\end{abstract}

Keywords: Line segment Hausdorff distance; Hausdorff distance; Line segment; Structure; Disparity; Face recognition

\section{Introduction}

Psychological studies $[1,2]$ indicated that human recognizes line drawings as quickly and almost as accurately as gray level pictures. These results might implicate that edge images of objects can be used for object recognition and achieve similar accuracy as gray level images. Takács [3] used Hausdorff distance to measure the similarity of two binary frontal face images, and achieved $92 \%$ accuracy in identifying the input. He argued that the process of face recognition may start at a much earlier stage and edge images can be used for fast screening of faces without the involvement of high-level cognitive functions. Olson and Huttenlocher [4] proposed an oriented Hausdorff distance that makes use of the direction of gradient to cut down the errors of false positives. The use of orientation information improves the recognition performance for dense and complicated object matching. Based on these studies, a novel object recognition approach is proposed here to harness the structural and spatial information of an object edge map. Edge images have the

\footnotetext{
* Corresponding author. Tel.: + 65-790-4319; fax: + 65-7926559 .

E-mail addresses: asysgao@ntu.edu.sg (Y. Gao), asmkleung@ntu.edu.sg (M.K.H. Leung).
}

advantage of less demand on storage space and they are less sensitive to illumination changes. After thinning of the edge map, a polygonal line fitting process is applied to generate the line segment representation of an object. This representation, using dominant points (i.e. end points of line segments) on the curves, further reduces the storage requirement. The proposed line segment Hausdorff distance (LHD) measure is then employed to match objects. Compared to conventional applications of Hausdorff distance, LHD has better distinctive power because it can make uses of the additional attributes of line orientation and line-point association, i.e. it is not encouraged to match two lines with large orientation difference, and all the points on one line have to match to points on another line only. The proposed technique has been applied on line segments generated from the edge maps of faces for face identification. Encouraging results that support the concept experimentally were obtained. The results also implicate that line segments could provide sufficient information for face recognition. This might imply a new way for face coding and recognition.

In the following, a brief introduction of Hausdorff distance together with a discussion on its weaknesses is highlighted in Section 2. In Section 3, a novel line segment Hausdorff distance for line matching is proposed and described in detail. Encouraging experimental 
results on human face recognition are reported in Section 4. Finally, the paper is concluded in Section 5.

\section{Hausdorff distance}

The Hausdorff distance is a shape comparison metric based on binary image. It is a distance measure defined between two point sets. Unlike most shape comparison methods that build a one-to-one correspondence between a model and a test image, Hausdorff distance can be calculated without explicit point correspondence [5]. Huttenlocher et al. [6] argued that the Hausdorff distance for binary image matching is more tolerant to perturbations in the locations of points than binary correlation techniques since it measures proximity rather than exact superposition. On the other hand, the images to be matched should be roughly aligned.

Given two finite point sets $M^{p}=\left\{m_{1}^{p}, m_{2}^{p}, \ldots, m_{k}^{p}\right\}$ (representing a model in the database) and $T^{p}=$ $\left\{t_{1}^{p}, t_{2}^{p}, \ldots, t_{n}^{p}\right\}$ (representing a test image) where the superscript $p$ stands for point, the Hausdorff distance is defined as

$H\left(M^{p}, T^{p}\right)=\max \left(h\left(M^{p}, T^{p}\right), h\left(T^{p}, M^{p}\right)\right)$,

where

$h\left(M^{p}, T^{p}\right)=\max _{m_{i}^{p} \in M^{p}} \min _{t_{j}^{p} \in T^{p}}\left\|m_{i}^{p}-t_{j}^{p}\right\|$

and $\left\|m_{i}^{p}-t_{j}^{p}\right\|$ is some underlying norm on the points of $M^{p}$ and $T^{p}$ (e.g. the $L_{2}$ or Euclidean norm). The function $h\left(M^{p}, T^{p}\right)$ is called the directed Hausdorff distance from $M^{p}$ to $T^{p}$ [6]. It identifies the point $m_{i}^{p} \in M^{p}$ that is the farthest from any point of $T^{p}$ and measures the distance from $m_{i}^{p}$ to its nearest neighbor in $T^{p}$. The Hausdorff distance $H\left(M^{p}, T^{p}\right)$ is the maximum of $h\left(M^{p}, T^{p}\right)$ and $h\left(T^{p}, M^{p}\right)$. Thus, it measures the degree of mismatch between two sets by measuring the distance of the point of $M^{p}$ that is farthest from any point of $T^{p}$ and vice versa [6].

Dubuisson and Jain [7] investigated 24 forms of different Hausdorff distance measures and indicated that a modified Hausdorff distance (MHD) measure gave the best performance. The directed MHD is defined as

$h\left(M^{p}, T^{p}\right)=\frac{1}{N_{m}^{p}} \sum_{m_{i}^{p} \in M^{p}} \min _{t_{j}^{p} \in T^{p}}\left\|m_{i}^{p}-t_{j}^{p}\right\|$,

where $N_{m}^{p}$ is the number of points in $M^{p}$. The definition of undirected MHD is the same as Eq. (1). The Hausdorff distance defined as Eqs. (1) and (2) is very sensitive to outlier points. A few outlier points, even only a single one, can perturb the distance greatly, though the two objects might be very similar. The MHD is less sensitive

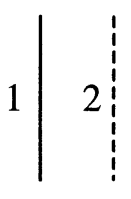

(a)

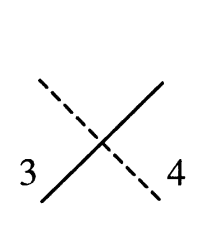

(b)

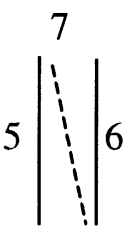

(c)
Fig. 1. Examples of matching problems using MHD. Solid lines represent the line segments in the model and dashed lines represent the line segments in the test image.

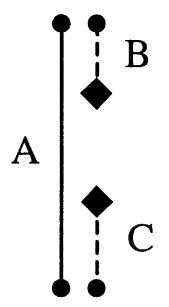

Fig. 2. An example of matching problem on broken line using MHD.

to outlier points that might result from segmentation errors.

In general, MHD still suffers from the following weaknesses. First, it uses spatial information of an image but lacks of local structure representation such as the orientation information. Two examples are illustrated in Fig. 1(a) and (b) where lines 1 and 2 are parallel while lines 3 and 4 are perpendicular. Unfortunately, the MHD measures of (a) and (b) can be the same but the lines from (a) are judged to be more similar by human observers. Fig. 1(c) shows another example where the points on the upper part of line 7 match to line 5 while those on the lower part match to line 6 . Intuitively, line 7 should be seen as one entity and be matched to either line 5 or 6 . These types of mismatches can be crucial in some matching problems, such as human face recognition, because the difference is minor from face to face (they are all within the class of human face). Another concern is about the broken lines caused by illumination variation and segmentation error as illustrated in Fig. 2. They can increase the MHD measure and cause mismatches.

In order to deal with these problems, a novel line segment Hausdorff distance (LHD) is proposed here to extend the MHD measure to match two sets of line segments. LHD makes uses of two new attributes of line orientation and line-point association. On the other hand, it needs to overcome some problems caused by using lines. The technical detail of LHD would be described in the next section. 


\section{Line segment Hausdorff distance}

Given two finite line segment sets $M^{l}=$ $\left\{m_{1}^{l}, m_{2}^{l}, \ldots, m_{p}^{l}\right\}$ (representing a model in the database) and $T^{l}=\left\{t_{1}^{l}, t_{2}^{l}, \ldots, t_{q}^{l}\right\}$ (representing a test image) where the superscript $l$ stands for line, LHD is built on the vector $\vec{d}\left(m_{i}^{l}, t_{j}^{l}\right)$ that represents the distance between two line segments $m_{i}^{l}$ (in the model) and $t_{j}^{l}$ (in the test image). The vector is defined as

$\vec{d}\left(m_{i}^{l}, t_{j}^{l}\right)=\left[\begin{array}{l}d_{\theta}\left(m_{i}^{l}, t_{j}^{l}\right) \\ d_{\|}\left(m_{i}^{l}, t_{j}^{l}\right) \\ d_{\perp}\left(m_{i}^{l}, t_{j}^{l}\right)\end{array}\right]$,

where $d_{\theta}\left(m_{i}^{l}, t_{j}^{l}\right), d_{\|}\left(m_{i}^{l}, t_{j}^{l}\right), d_{\perp}\left(m_{i}^{l}, t_{j}^{l}\right)$ are the angle distance, parallel distance and perpendicular distance, respectively.

All these three entries are independent and defined as

$d_{\theta}\left(m_{i}^{l}, t_{j}^{l}\right)=f\left(\theta\left(m_{i}^{l}, t_{j}^{l}\right)\right)$,

$d_{\|}\left(m_{i}^{l}, t_{j}^{l}\right)=\min \left(l_{\| 1}, l_{\| 2}\right)$,

$d_{\perp}\left(m_{i}^{l}, t_{j}^{l}\right)=l_{\perp}$,

where $\theta\left(m_{i}^{l}, t_{j}^{l}\right)$ computes the smallest intersecting angle between lines $m_{i}^{l}$ and $t_{j}^{l}$. $f()$ is a non-linear penalty function to map the angle to a scalar. It is desirable to ignore small angle variation but penalize heavily on large deviation. In this study, the tangent function is used. An upper limit of $\tan 89^{\circ}$ is set for $d_{\theta}\left(m_{i}^{l}, t_{j}^{l}\right)$ to avoid calculation of $\tan 90^{\circ}$. The designs of the parallel and perpendicular displacements can be illustrated with a simplified example of two parallel lines, $m_{i}^{l}$ and $t_{j}^{l}$, as shown in Fig. 3. $d_{\|}\left(m_{i}^{l}, l_{j}^{l}\right)$ is defined as the minimum displacement to align either the left end points or the right end points of the lines. $d_{\perp}\left(m_{i}^{l}, t_{j}^{l}\right)$ is simply the vertical distance between the lines. In general, $m_{i}^{l}$ and $t_{j}^{l}$ would not be parallel but one can rotate the shorter line with its midpoint as rotation center to the desirable orientation before computing $d_{\|}\left(m_{i}^{l}, t_{j}^{l}\right)$ and $d_{\perp}\left(m_{i}^{l}, t_{j}^{l}\right)$. Fig. 4 shows 3 typical rotations. The purpose of rotation is to calculate $d_{\|}\left(m_{i}^{l}, t_{j}^{l}\right)$ and $d_{\perp}\left(m_{i}^{l}, t_{j}^{l}\right)$. Among the three rotations of Figs. 4(b), (c) and (d), (b) is found to give measures of $d_{\|}\left(m_{i}^{l}, t_{j}^{l}\right)$ and $d_{\perp}\left(m_{i}^{l}, t_{j}^{l}\right)$ closest to the original values by human observers. In order to cater for the effect of broken lines caused by segmentation error, and alleviate the effect of adding, missing and shifting of feature points (i.e. end points of line segments) caused by inconsistency of feature point detection, the parallel shifts $l_{\| 1}$ and $l_{\| 2}$ are reset to zero if one line is within the range of the other as shown in Fig. 5. Finally, the distance between two line segments $m_{i}^{l}$ and $t_{j}^{l}$ is defined as

$d\left(m_{i}^{l}, t_{j}^{l}\right)=\sqrt{\left(W_{a} \cdot d_{\theta}\left(m_{i}^{l}, t_{j}^{l}\right)\right)^{2}+d_{\|}^{2}\left(m_{i}^{l}, t_{j}^{l}\right)+d_{\perp}^{2}\left(m_{i}^{l}, t_{j}^{l}\right)}$,

where $W_{a}$ is the weight for angle distance and would be determined in the next section. The directed and undirec-

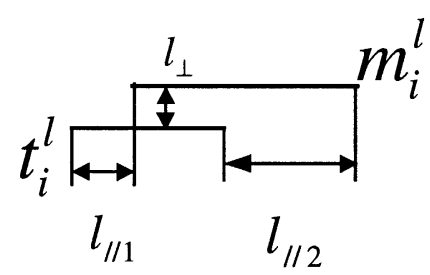

Fig. 3. Line displacement measures. (a)

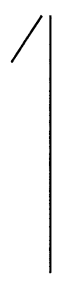

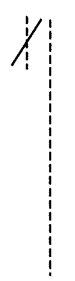

(b)

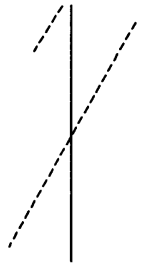

(c)

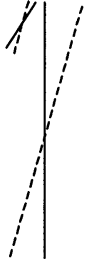

(d)
Fig. 4. The rotation effect of two lines. (a) The original line pair. (b) Rotate the shorter line. (c) Rotate the longer line. (d) Rotate both lines with half of their angle difference in opposite directions. Solid lines represent lines before rotation. Dashed lines represent lines after rotation.

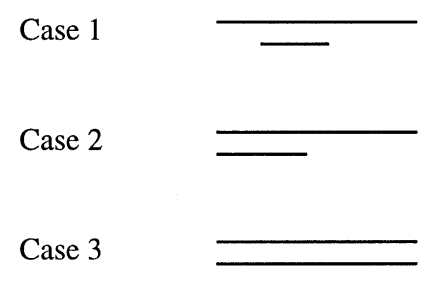

Fig. 5. All cases with $d_{\|}(m, t)=0$.

ted LHDs are defined in Eqs. (8) and (9), as

$$
\begin{aligned}
& h_{l}\left(M^{l}, T^{l}\right)=\frac{1}{\sum_{m^{l} \in M^{l}} l_{m_{i}^{l}}} \sum_{m_{i}^{l} \in M^{l}} l_{m_{i}^{l}} \cdot \min _{t_{j}^{l} \in T^{l}} d\left(m_{i}^{l}, t_{j}^{l}\right), \\
& H_{l}\left(M^{l}, T^{l}\right)=\max \left(h_{l}\left(M^{l}, T^{l}\right), h_{l}\left(M^{l}, T^{l}\right)\right),
\end{aligned}
$$

where $l_{m_{i}^{l}}$ is the length of line segment $m_{i}^{l}$.

\section{Experimental results}

The proposed LHD was compared to MHD in the following experiments to evaluate their performance on human frontal face/profile matching, face recognition under varying lighting condition and expression. Preprocessing was needed to align inputs with the models. The eye positions were used for frontal faces while the distance from nose tip to chin point was used for profile faces in the alignment process. The face database from the University of Bern [11] was used to test the system 


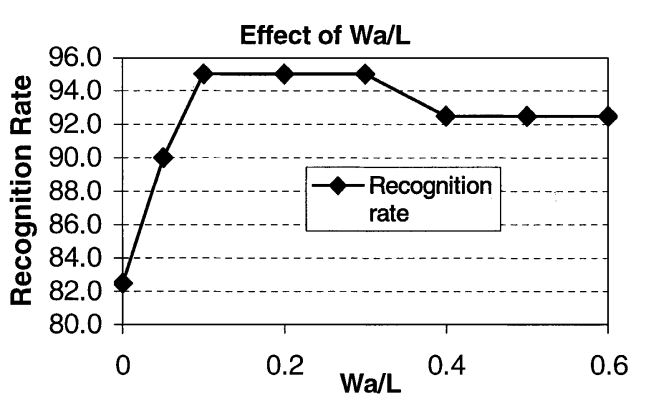

Fig. 6. The effect of $W_{a}$ on recognition of misaligned profiles.

performances on frontal faces, profile faces, profile outlines and misaligned profile faces. AR face database [8] of Purdue University was used to investigate effects of varying lighting condition and facial expression, while the Yale face database [9] was tested in order to compare LHD and MHD to the reported results of Eigenface approach. LHD performed consistently superior to MHD experimentally.

\subsection{The effect of $W_{a}$}

The effect of $W_{a}$ in Eq. (7) was investigated using misaligned profile faces (Fig. 10) in order to choose a value of $W_{a}$ which provides LHD a better tolerance to alignment errors. The misalignments were simulated by shifting the two end points of $L$ by a few pixels [10] where $L$ is the distance between nose tip and chin point. Twenty people with 2 profiles per person in the database were used. The recognition rate is plotted against the normalized $W_{a}$ (i.e. $W_{a} / L$ ) in Fig. 6. It is found that LHD without the orientation information (i.e. $W_{a}=0$ ) performed worse than MHD (87.5\% in Table 1). It improved quickly with $W_{a} / L=0.1$ and reached the optimal value when $W_{a} / L$ ranged from 0.1 to 0.3 . For all the other experiments in this study, $W_{a}$ was set as 20 (i.e. $\left.W_{a} / L=0.2\right)$.

\subsection{Face recognition}

A face database of 30 persons with 2 profiles and 2 frontal images per person from the University of Bern [11] was used to test the capability of the proposed LHD approach. Each image has the size of $512 \times 342$ pixels with 256 gray levels and variations of the head position, size and contrast. One profile and one frontal face images of each person were used as models while the others were used as inputs. Though there are only 30 pairs of models and inputs, one can have 60 matching experiments if the roles of model and input are interchanged. The nose tip and chin point for each profile face were detected by the system automatically. The distance, $L$, between these two points was used as a reference to normalize image size, align face position and cut out facial area to avoid processing subject's hair. For the frontal face, the eye locations were detected manually for normalization, alignment and cutting. Sample pairs of the frontal and the profile face edge maps (models and test images) together with the detected dominant points [12] are shown in Figs. 7 and 8. Note that there are instances of broken lines, dense edges and adding/missing/shifting of feature points from one image to its match.

The recognition results on faces are summarized in Table 1 while the legends of symbols are displayed in Table 2. Four types of data were tested. They were the frontal faces, profile faces, profile outlines (Fig. 9), and misaligned profile faces (Fig. 10). It is found that LHD scores equally well or better in all experiments (Table 1, row 3). Note that the least score of LHD is $95 \%$.

For the profile faces, LHD achieved a recognition rate of $96.7 \%$. The only case of mismatch happened to the person whose hair area showed great difference from the input to the model (Fig. 8(d)). They matched best to a person with similar hairstyle (Fig. 8(c)). This error can be overcome by proper preprocessing. For the misaligned profiles, the recognition rate of LHD was remarkably higher than MHD by $7.5 \%$. This demonstrates that the added structural information makes LHD robust to image alignment errors.

Table 1

Face recognition results of MHD and LHD with $W_{a}=20$

\begin{tabular}{|c|c|c|c|c|c|c|c|c|}
\hline \multirow{2}{*}{$\begin{array}{l}\text { Data } \\
\text { Method }\end{array}$} & \multicolumn{2}{|c|}{ Profile outlines } & \multicolumn{2}{|l|}{ Profiles } & \multicolumn{2}{|c|}{ Frontal faces } & \multicolumn{2}{|c|}{ Misaligned profiles } \\
\hline & MHD & LHD & MHD & LHD & MHD & LHD & MHD & LHD \\
\hline$R$ & $93.3 \%$ & $96.7 \%$ & $96.7 \%$ & $96.7 \%$ & $96.7 \%$ & $100 \%$ & $87.5 \%$ & $95 \%$ \\
\hline$|\vec{D}|$ & 4.09 & 4.80 & 4.66 & 6.06 & 2.85 & 4.00 & 3.98 & 5.35 \\
\hline$D_{d}$ & 4.09 & 3.50 & 4.66 & 4.37 & 2.85 & 2.78 & 3.98 & 3.72 \\
\hline$D_{o}$ & NA & 3.28 & NA & 4.20 & NA & 2.88 & NA & 3.84 \\
\hline$\left|\overrightarrow{\bar{d}}^{\text {all }}\right|$ & 5.30 & 7.93 & 6.82 & 10.99 & 4.72 & 8.59 & 6.70 & 10.80 \\
\hline$\left|\vec{d}^{c o r}\right|$ & 1.29 & 3.20 & 2.16 & 5.05 & 1.87 & 4.68 & 2.72 & 5.50 \\
\hline
\end{tabular}




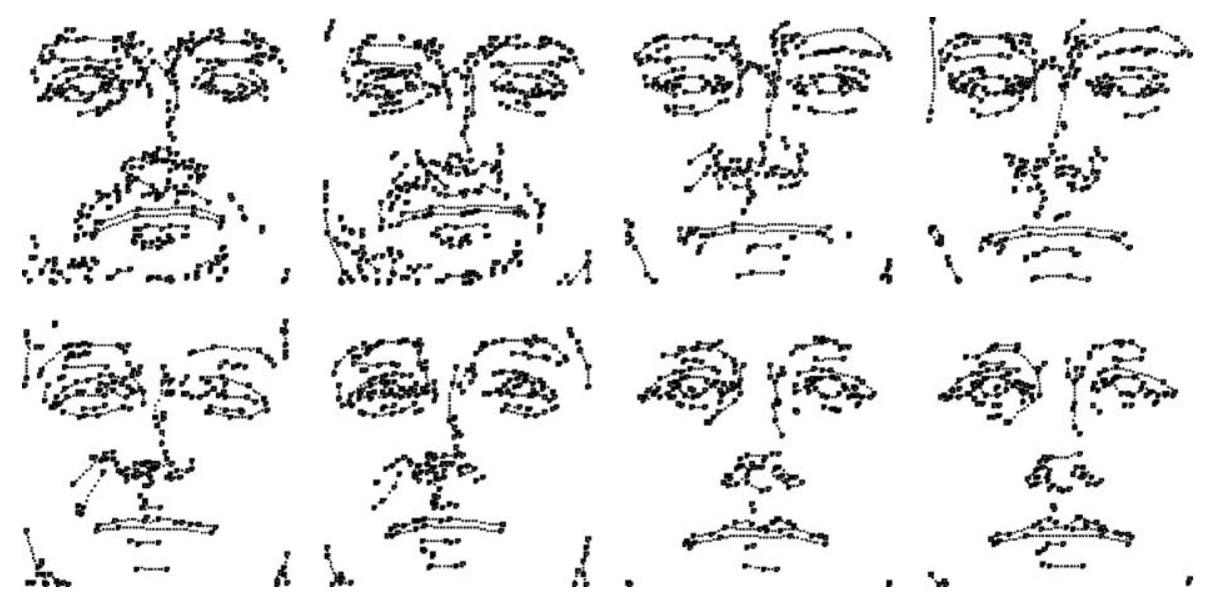

Fig. 7. Sample pairs of frontal face edge maps with detected dominant points.

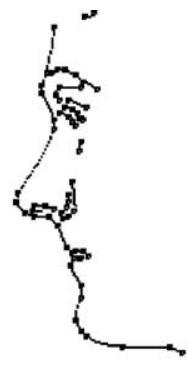

(a)
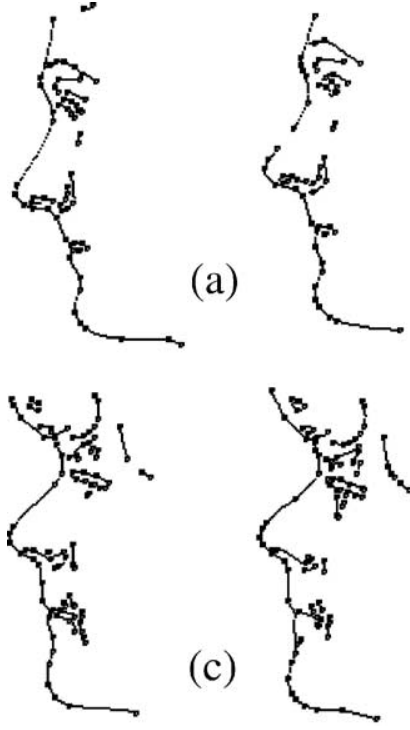

(c)

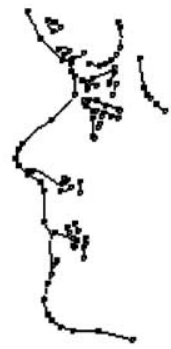

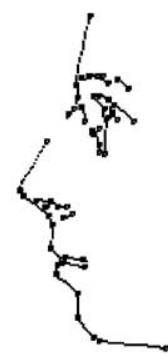

(b)
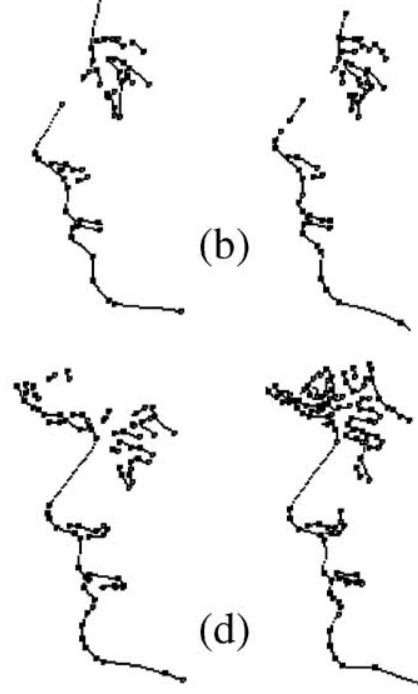

(d)

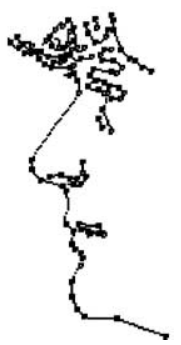

Fig. 8. Sample pairs of profile edge maps with detected dominant points.

Table 2

Legends of symbols in Table 1

\begin{tabular}{ll}
\hline$R$ & Recognition rate \\
$|\vec{D}|$ & Disparity $\left(\left|\vec{d}^{\text {all }}-\vec{d}^{\text {cor }}\right|\right)$ \\
$D_{d}$ & Displacement disparity $\left(\vec{d}_{d}^{\text {all }}-\vec{d}_{d}^{\text {cor }}\right)$ \\
$D_{o}$ & Orientation disparity $\left(\vec{d}_{o}^{\text {all }}-\vec{d}_{o}^{\text {cor }}\right)$ \\
$\left|\vec{d}^{\text {all }}\right|$ & Average distance of all matches \\
$\left|\vec{d}^{\text {cor }}\right|$ & Average distance of the correct matches \\
\hline
\end{tabular}

Let $\vec{d}^{c o r}$ be the average distance vector of the correct matches and $\vec{d}^{\text {all }}$ be the average distance vector of all matches as shown in Fig. 11. The disparity vector, $\vec{D}$, which is the difference of $\vec{d}^{\text {all }}$ and $\vec{d}^{\text {cor }}$, can be used as another measure to compare system recognition capability. It is desirable to have large $\vec{D}$ value. The scalar values of $\vec{D},|\vec{D}|$, are displayed in row 4 of Table 1 . It can be seen that LHD has a much higher value on all four experiments. The projections of $\vec{D}$ along the directions of displacement and orientation are shown in rows 5 and 6 . It is observed that LHD and MHD share similar values in row 5. This suggests that the proposed LHD displacement computation, $D_{d}$, is comparable to the $|\vec{D}|$ (equal to $D_{d}$ ) of MHD. The increase of the disparity $|\vec{D}|$ for LHD with respect to MHD is due to the contribution of the new attribute of orientation distance $D_{o}$, which is independent of the displacement distance $D_{d}$. As the $D_{d}$ of LHD is comparable to the disparity $(|\vec{D}|)$ of MHD, 

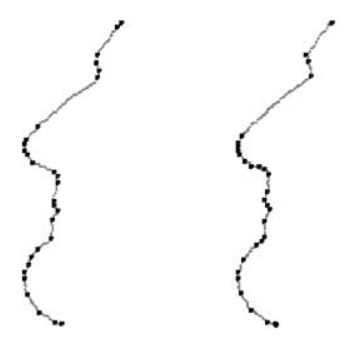

pair 1

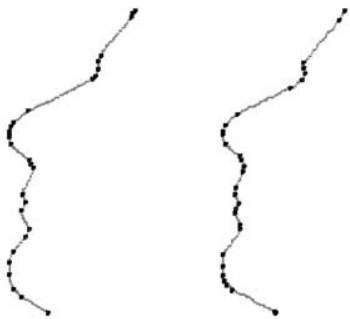

pair 2
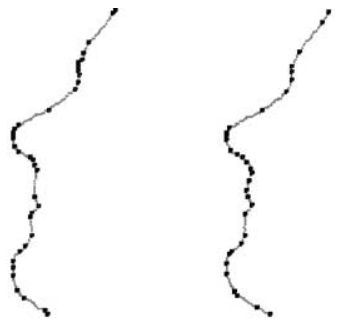

pair 3
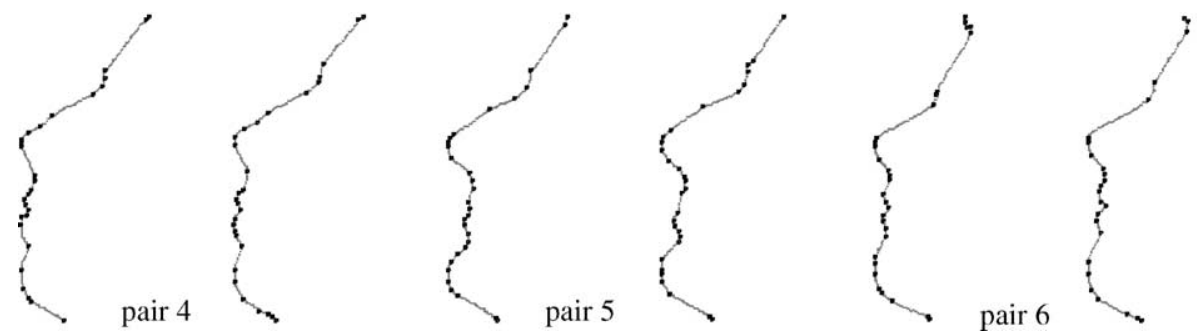

Fig. 9. Sample pairs of face profiles together with the detected dominant points.

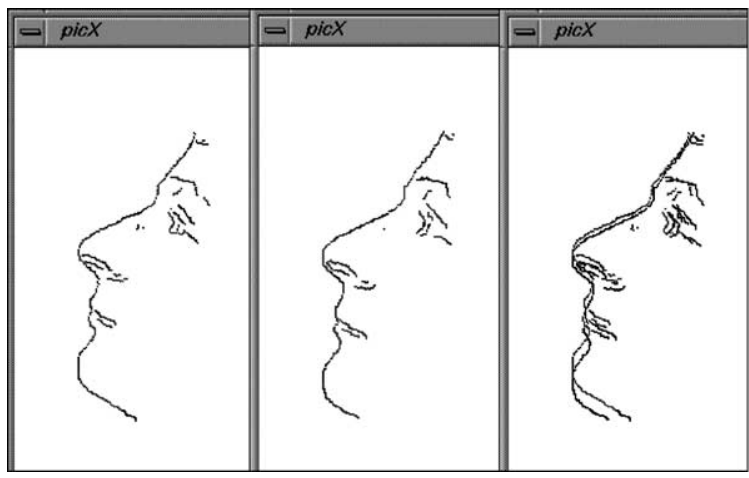

Fig. 10. An example of misaligned model and input faces.

the disparity $|\vec{D}|$ of LHD should have a greater discriminating power than the 1D disparity of MHD. This is indeed reflected in row 4 of Table 1 and serves to demonstrate that LHD is a more discriminative measure than MHD.

\subsection{Face recognition with lighting changes}

To evaluate the performance of LHD and MHD to lighting condition variations, an experiment was designed and applied on face images with neutral expression taken under different lighting conditions from Purdue University [14]. The faces in neutral expression under background illumination (the upper left image of Fig. 12) were used as single models of the subjects. The images under three different lighting conditions (light from the left, right and both directions) were used as test

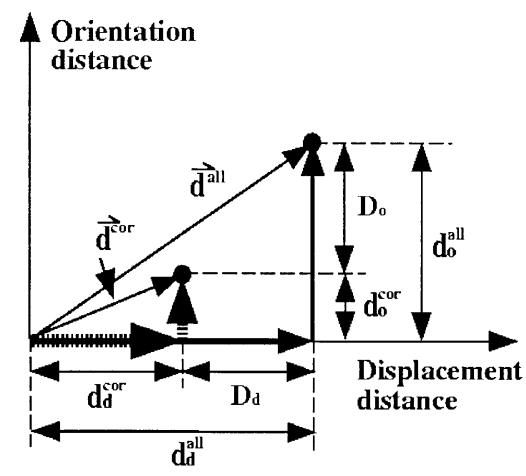

Fig. 11. Illustration of average LHD composition.

images (third row of Fig. 12). There are totally 112 models (representing 112 individuals) and 336 test images. Other 112 images in neutral expression under background illumination taken after 14 days were used to generate a performance benchmark without lighting condition changes.

The experimental results under three different lighting conditions are illustrated in Figs. 13, 14 and 15, respectively, while the recognition results for faces taken after 2 weeks without lighting condition and facial expression changes are shown in Fig. 16.

These experiments reveal a number of interesting points:

1. In all the four experiments, LHD consistently performed better than MHD with an improvement of 5-7\% in recognition rate. 


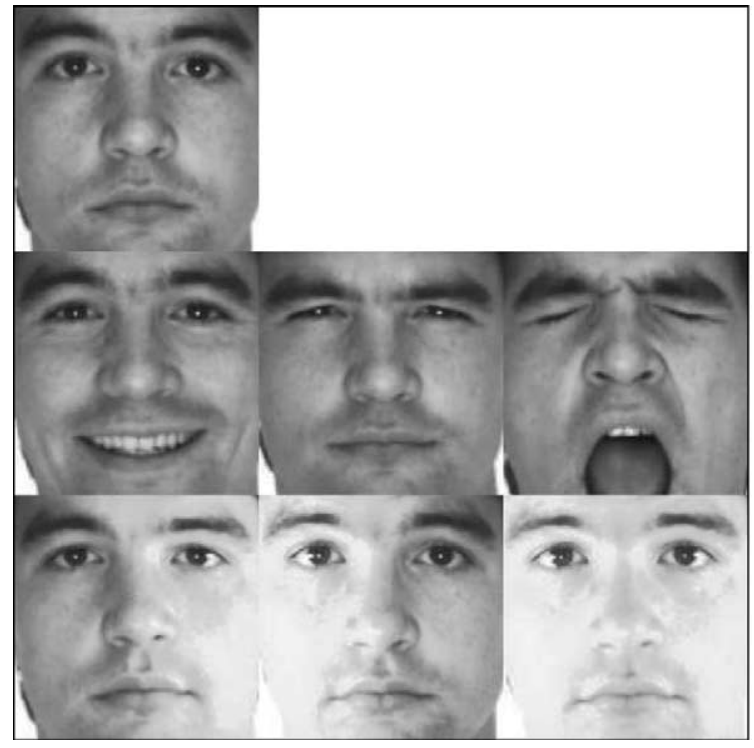

Fig. 12. Sample cropped images of model (first row) and test faces (second and third rows).

\section{Recogntion rate}

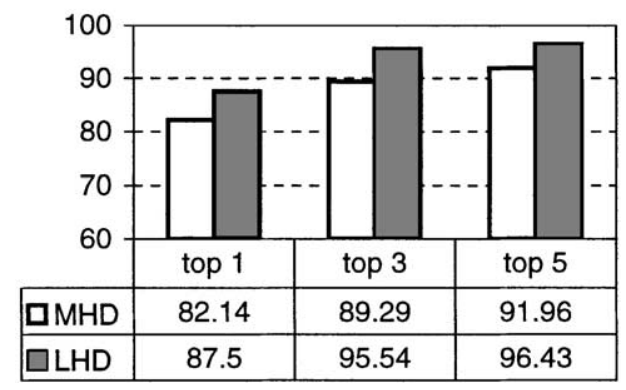

Fig. 13. Recognition results when left light was on.

2. The variations of lighting condition did affect the system performance. Nevertheless, the recognition rates when one light was on were still high.

3. When both lights were on, the error rates became much higher than that of only one light was on. This evidence shows that the edge map would still be affected by extreme lighting condition variations, such as over-illumination, though it is insensitive to some extent.

4. It was found that the recognition rates with the left light on were higher than that with the right light on. This could be caused by the fact that the illumination on a face from the right light was actually stronger than that from the left light.

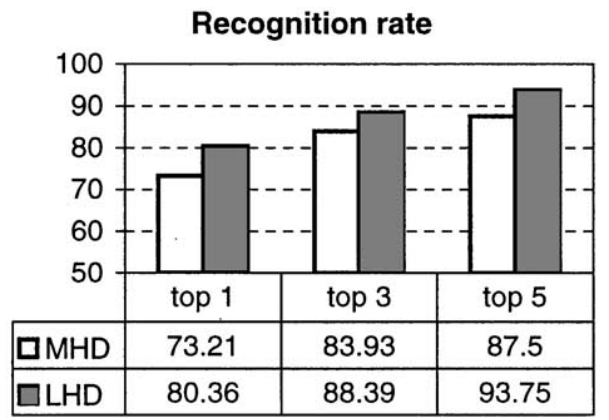

Fig. 14. Recognition results when right light was on.

\section{Recognition rate}

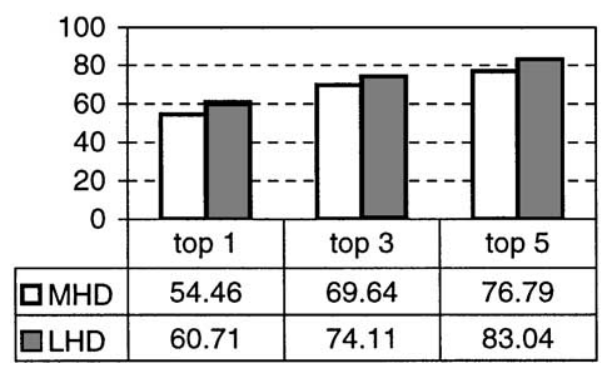

Fig. 15. Recognition results when both lights were on.

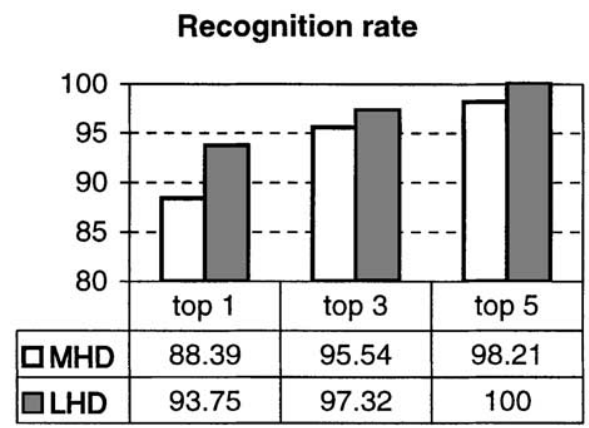

Fig. 16. Recognition results with background lighting after two weeks.

\subsection{Face recognition under facial expression changes}

Similar experiments were conducted to evaluate the effects of different facial expressions (smile, anger and scream) on the system performance. The face images of different facial expressions in the face database of Purdue University were used in the experiments. The face in neutral expression was used as the single model of the subject. The images in smile, anger and scream expressions were used as test images. There are totally 112 


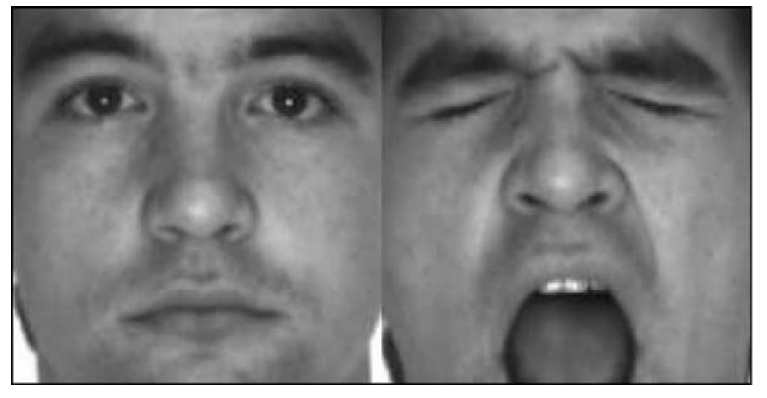

Fig. 17. An illustration of large variation between the model and the scream expression face.

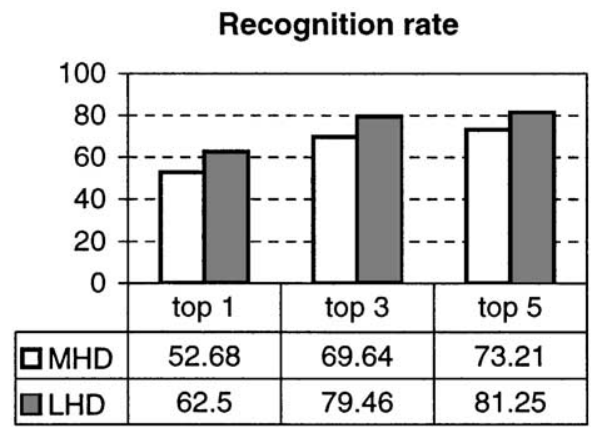

Fig. 18. Recognition results on smiling expression.

\section{Recognition rate}

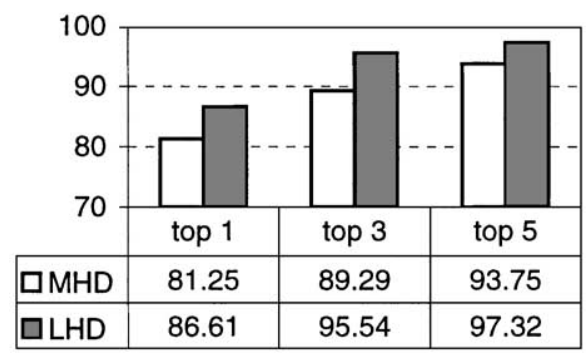

Fig. 19. Recognition results on angry expression.

models (representing 112 individuals) and 336 test images.

The experimental results on faces with smile, anger and scream expressions (second row of Fig. 12) were summarized in Figs. 18, 19 and 20, respectively. They illustrate that the smile expression has caused the recognition rate to drop from $17.86 \%$ to $35.71 \%$ as compared to Fig. 16. On the other hand, the anger expression causes only $3.57-7.14 \%$ drop of rate. This is not unexpected because the anger expression produces less physical variation from the neutral expression. The scream expression could

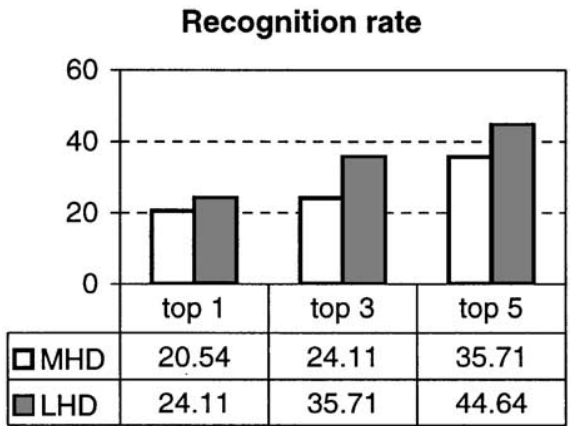

Fig. 20. Recognition results on screaming expression.

be the extreme case of deformation among various human facial expressions, i.e. most of the facial features have been distorted (see Fig. 17). The variations between images from the same face are larger than that from different faces. The experimental result (Fig. 20) on this extreme facial expression is very interesting. Instead of totally failure to identify the input, LHD can still identify $24.11 \%$ of the input. This is much higher than the random probability of $0.89 \%$ (i.e. pick one out of 112 models). Again, LHD performs better than MHD by $3.5-9.8 \%$ in all three experiments.

\subsection{Leave-one-out test}

Belhumeur et al. [13] designed tests to investigate how different face recognition methods compared under a different range of conditions using the Yale face database [9]. The database, constructed at the Yale Centre for Computational Vision and Control, is composed of 165 images of 15 subjects. The images demonstrate variations in lighting condition (left-light, center-light, right-light), facial expression (normal, happy, sad, sleepy, surprised, and wink) and with/without glasses. Fig. 21 shows 11 images of one subject. Experiments were performed using a "leaving-one-out" strategy: the models are constructed with all images of subjects except the one used as input.

The same strategy was adopted here in order to make comparisons of LHD, MHD and the well-known Eigenface approach by using the same face database. For comparison purpose, the images were similarly processed as stated by Belhumeur et al. to generate closely cropped images that include internal structures such as the eyebrows, eyes, nose and mouth, but did not extend to the occluding contour. The result cannot be precisely compared because the facial area cut into the cropped face by Belhumeur et al. may be slightly different from those used in this study. Here we intentionally excluded the chins from the cropped faces (Fig. 21) though they were included in the cropped faces by Belhumeur et al. 


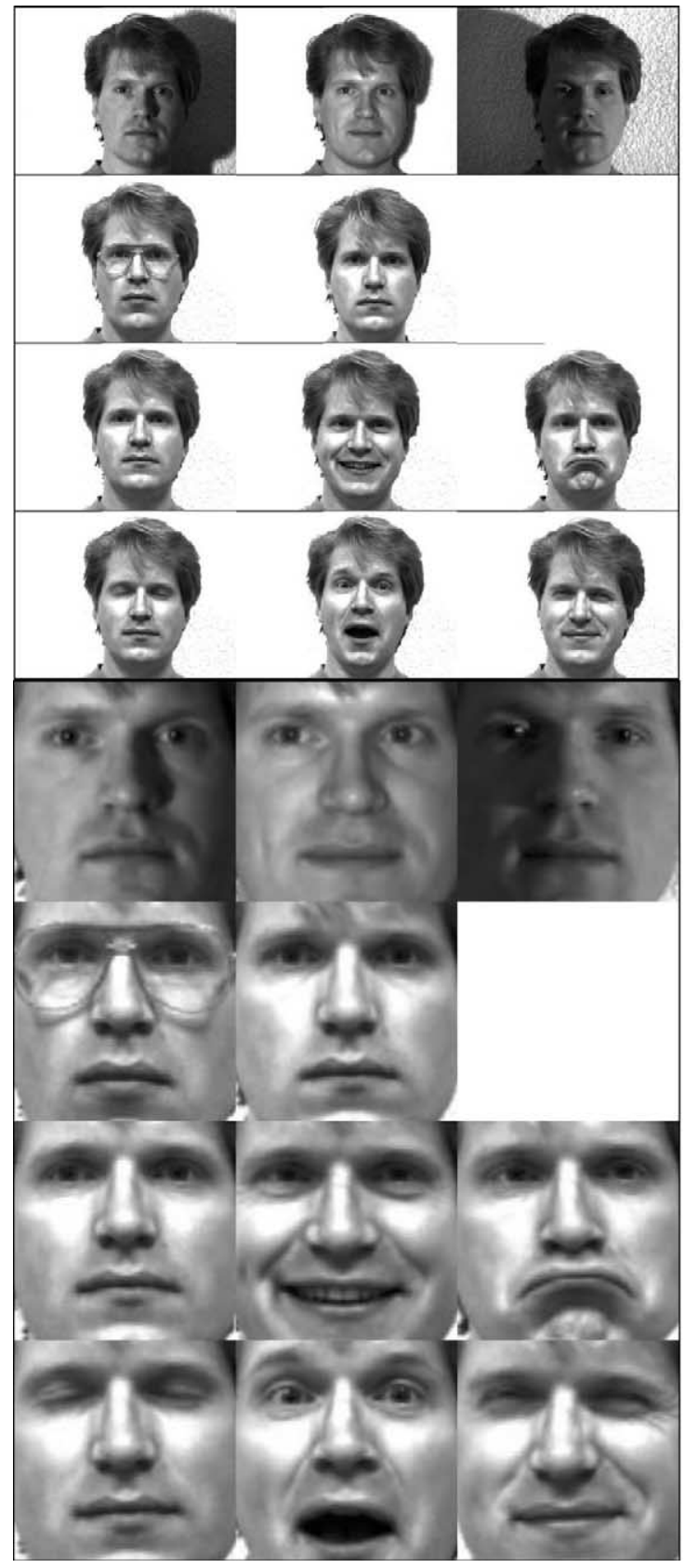

Fig. 21. The Yale face database contains 165 frontal face images covering 15 individuals taken under 11 different conditions. The faces in the first 4 rows are original images while the last 4 rows show the cropped faces used in our experiment.

A rough comparison of the error rates show that the LHD performed better by $2.6 \%$ higher recognition rate than Eigenface while MHD obtained 1.7\% higher error rate than Eigenface (Table 3). This indicates that LHD performs slightly superior to Eigenface method for
Table 3

"Leave-one-out" test of Yale face database

\begin{tabular}{ll}
\hline Method & Error rate $(\%)$ \\
\hline MHD & 26.1 \\
Eigenface $^{\mathrm{a}}$ & 24.4 \\
LHD & 21.8 \\
\hline
\end{tabular}

${ }^{\mathrm{a}}$ The values are from Ref. [13].

identifying faces under varying lighting condition and expression while the performance of MHD is inferior to Eigenface.

\subsection{Storage requirement and computational complexity}

The storage requirement of LHD is analyzed based on the two profile and frontal face databases from the Bern University. The data sizes in the experiments are listed in Table 4. On average, a profile face can be represented by 78 feature points and a frontal face can be coded with 293 feature points. Compared with the edge map that MHD used, LHD requires only $27.3 \%$ and $28.5 \%$ storage space for the profile and the frontal face, respectively. This is an important improvement of LHD over MHD especially for large database identification applications.

The computational complexities of LHD and MHD are of the order of $O\left(k_{l} N_{m}^{l} N_{t}^{l}\right)$ and $O\left(k_{p} N_{m}^{p} N_{t}^{p}\right)$, respectively. $N_{m}^{l}$ and $N_{t}^{l}$ are the line segment numbers of model and test faces while $N_{m}^{p}$ and $N_{t}^{p}$ are the pixel numbers of model and test edge maps. $k_{l}$ and $k_{p}$ are the time to compute $d\left(m_{i}^{l}, t_{j}^{l}\right)$ of (7) in LHD and the Euclidean norm $\left\|m_{i}^{p}-t_{j}^{p}\right\|$ in MHD. Table 5 shows the average real computation time of MHD and LHD on profile and frontal faces. The experiments were conducted on a SGI Octane workstation with $300 \mathrm{MHz}$ CPU and 512MB RAM. The computational time of LHD is less than $50 \%$ of that of MHD. LHD took $0.051 \mathrm{~s}$ for one profile match and $0.502 \mathrm{~s}$ for one frontal face match.

\section{Conclusion}

A novel object matching approach (LHD), which employs both spatial and structural information of edge map, is proposed in this paper. The major contribution of LHD is that it takes line segments rather than pixels as basic unit into computation with the following interesting properties and findings:

1. LHD incorporates structural information of line orientation and line-point association.

2. LHD demands much less storage space than MHD and therefore requires less computational demand.

3. Both LHD and MHD have shown encouraging experimental results with high recognition rate but LHD 
Table 4

Average storage requirements of profile and frontal faces in the experiments

\begin{tabular}{lll}
\hline & $\begin{array}{l}\text { Pixel number } \\
\text { (for MHD matching) }\end{array}$ & $\begin{array}{l}\text { Feature point number } \\
\text { (for LHD matching) }\end{array}$ \\
\hline Profile & 287 & 78 \\
Frontal face & 1027 & 293 \\
\hline
\end{tabular}

Table 5

Average computational time of MHD and LHD

\begin{tabular}{lll}
\hline & MHD (sec.) & LHD (sec.) \\
\hline Profile & 0.109 & 0.051 \\
Frontal face & 1.146 & 0.502 \\
\hline
\end{tabular}

performed equally well or better than MHD on all the test data.

4. LHD degrades gracefully with misaligned images as compared to MHD because LHD makes use of structural information. This can alleviate the difficulty of image alignment in object matching since alignment is still an unsolved problem in many applications.

In this experiment, LHD performs slightly superior to Eigenface method for identifying faces under lighting condition and expression changes while the performance of MHD is inferior to Eigenface. The experimental results demonstrated that line segment of human faces could provide sufficient information for face recognition. This might provide a new way for face coding and recognition. Nevertheless, a number of research issues need to be addressed in the future. In particular, efficient matching algorithm of LHD such as the distance transform, which had been used for reducing the computational complexity of Hausdorff distance measure, should be investigated. Other existing face recognition methods should be implemented and tested using the same face databases in order to make a direct comparison.

\section{References}

[1] I. Biederman, J. Gu, Surface versus edge-based determinants of visual recognition, Cognitive Psychol. 20 (1988) $38-64$.

[2] V. Bruce et al., The importance of 'Mass' in line drawings of faces, Appl. Cognitive Psychol. 6 (1992) 619-628.

[3] B. Takács, Comparing face images using the modified Hausdorff distance, Pattern Recognition 31 (1998) 1873-1881.

[4] C.F. Olson, D.P. Huttenlocher, Recognition by matching dense, oriented edge pixels, Proceedings of the IEEE International Conference on Computer Vision, 1995, pp. 91-96.
[5] W.J. Ruchlidge, Efficiently locating objects using the Hausdorff distance, Int. J. Comput. Vision 24 (1997) 251-270.

[6] D.P. Huttenlocher, G.A. Klanderman, W.J. Rucklidge, Comparing images using the Hausdorff distance, IEEE Trans. Pattern Anal. Mach. Intell. 15 (1993) 850-863.

[7] M.P. Dubuisson, A.K. Jain, A modified Hausdorff distance for object matching, Proceedings 12th International Conference on Pattern Recognition, Jerusalem, Israel, 1994, pp. 566-568.

[8] Purdue University face database. http://rvl1.ecn.purdue.edu/ aleix/aleix_face_DB.html

[9] Yale University Face Database. http://cvc.yale.edu/projects/yalefaces/yalefaces.html

[10] Y.Gao, Human face recognition using line edge information, Annual Report, Nanyang Technological University, Singapore, 1999.

[11] University of Bern face database. ftp://iamftp.unibe.ch/ pub/Images/FaceImages/

[12] M.K. Leung, Y.H. Yang, Dynamic two-strip algorithm in curve fitting, Pattern Recognition 23 (1/2) (1990) 69-79.

[13] P.N. Belhumeur, J.P. Hespanha, D.J. Kriegman, Eigenfaces vs. fisherfaces: recognition using class specific linear projection, IEEE Trans. PAMI 19 (1997) 711-720.

[14] A. Martinez, R. Benavente, The AR face database, CVC Technical Report \#24, June, 1998.

\section{Further reading}

Y. Gao, M.K.H. Leung, Human face recognition using line edge maps, IEEE Second Workshop on Automatic Identification Advanced Technologies, New Jersey, USA, October 1999, pp. 173-176.

I.J. Cox, J. Ghosn, P.N. Yianios, Feature-based face recognition using mixture-distance, Computer Vision Pattern Recognition, IEEE Press, Piscataway, NJ, 1996.

Y. Gao, M.K.H. Leung, Recognize a rotated face from a single 2D model view, The Third International Conference on Computer Vision, Pattern Recognition and Image Processing, Atlantic City, NJ, USA, 2000.

R. Nevatia, K.R. Babu, Linear feature extraction and description, Comput. Graphics Image Process. 13 (1980) 257-269.

X. Yi, O.I. Camps, Line feature-based recognition using Hausdorff distance, Proceedings of the IEEE International Conference on Computer Vision, pp. 79-84, 1995.

H.G. Barrow, J.M. Tenenbaum, R.C. Bolles, H.C. Wolf, Parametric correspondence and chamfer matching: two new techniques for image matching, Proceedings of the Fifth International Joint Conference on Artificial Intelligence, 1977, pp. 659-663.

D.P. Huttenlocher, K. Kedem, Efficiently computing the Hausdorff distance for point sets under translation, Proceedings of the Sixth ACM Symposium on Computational Geometry, 1990, pp. 340-349.

G. Borgefors, Hierarchical chamfer matching: a parametric edge matching algorithm, IEEE Trans. Pattern Anal. Mach. Intell. 10 (1988) 849-865.

W.J. Rucklidge, Efficient computation of the minimum Hausdorff distance for visual recognition, Ph.D. Thesis, Cornell University, 1995. 
W.J. Rucklidge, Locating objects using the Hausdorff distance, Proceedings of the International Conference on Computer Vision, Cambridge, MA, 1995, pp. 457-464.

D.P. Huttenlocher, S. Ullman, Recognizing solid objects by alignment with an image, Int. J. Comput. Vision 5 (1990) 195-212.

M. Turk, A. Pentland, Eigenfaces for recognition, J. Cognitive Neurosci. 3 (1991) 71-86.
A. Pentland, B. Moghaddam, T. Starner, View-based and modular eigenspaces for Face recognition, Proceedings of the IEEE Computer Society Conference On Computer Vision and Pattern Recognition, 1994, pp. 84-91.

R. Bruneli, T. Poggio, Face recognition: features versus templates, IEEE Trans. Pattern Anal. Mach. Intell. 15 (1993) $1042-1052$.

About the Author-YONGSHENG GAO received the B.Sc. and M.Sc. degrees in Electronic Engineering from Zhejiang University, China, in 1985 and 1988, respectively, and his Ph.D. degree in Computer Engineering from Nanyang Technological University, Singapore. Currently, he is an assistant professor with Nanyang Technological University. His research interests include computer vision, pattern recognition and face recognition.

About the Author-MAYLOR K.H. LEUNG received the B.Sc. degree in Physics from the National Taiwan University in 1979, and the B.Sc., M.Sc. and Ph.D. degrees in Computer Science from the University of Saskatchewan, Canada, in 1983, 1985 and 1992, respectively. Currently, Dr. Leung is an associate professor with Nanyang Technological University, Singapore. His research interests include structural pattern processing, face recognition, motion analysis and string processing. 